\title{
Submicron Patterns-on-a-Chip: Fabrication of a Microfluidic Device Incorporating 3D Printed Surface Ornaments
}

\author{
Mahdiyeh Nouri-Goushki, ${ }^{*} \dagger, \nabla \odot$ Abhishek Sharma, ${ }^{\ddagger}, \nabla$ Luigi Sasso, ${ }^{\ddagger}$ Shuang Zhang, ${ }^{\S}$ \\ Bram C. J. Van der Eerden, ${ }^{\S}$ Urs Staufer, $^{\dagger}$ Lidy E. Fratila-Apachitei, ${ }^{\dagger}$ and Amir A. Zadpoor ${ }^{\dagger}$ \\ ${ }^{\dagger}$ Department of Biomechanical Engineering, Faculty of Mechanical, Maritime, and Materials Engineering, Delft University of \\ Technology (TU Delft), Mekelweg 2, 2628 CD Delft, The Netherlands \\ ${ }^{\ddagger}$ Department of Precision and Microsystems Engineering, Faculty of Mechanical, Maritime, and Materials Engineering, Delft \\ University of Technology (TU Delft), Mekelweg 2, 2628 CD Delft, The Netherlands \\ ${ }^{\S}$ Department of Internal Medicine, Erasmus Medical Center, Dr. Molewaterplein 40, 3015 GD Rotterdam, The Netherlands
}

\section{Supporting Information}

ABSTRACT: Manufacturing high throughput in vitro models resembling the tissue microenvironment is highly demanded for studying bone regeneration. Tissues such as bone have complex multiscale architectures inside which cells reside. To this end, engineering a microfluidic platform incorporated with three-dimensional (3D) microscaffolds and submicron/nanoscale topographies can provide a promising model for $3 \mathrm{D}$ cell cultures. There are, however, certain challenges associated with this goal, such as the need to decorate large surfaces area with high-fidelity 3D submicron structures. Here, we succeeded in fabricating a microfluidic platform embedded with a large area ( $\mathrm{mm}$ range) of reproducible submicron pillar-based topographies. Using the two-photon polymerization (2PP) as a 3D printing technique based on direct laser writing, uniform submicron patterns were created through optimization of the process parameters and writing strategy. To demonstrate the multiscale fabrication capabilities of this approach, submicron pillars of various heights were integrated onto the surfaces of a $3 \mathrm{D}$ microscaffold in a single-step $2 \mathrm{PP}$ process. The created submicron topography was also found to improve the hydrophilicity of the surface while being able to withstand flow rates of up to $8 \mathrm{~mL} / \mathrm{min}$. The material (IP-Dip resin) used for patterning did not have cytotoxic effects against human mesenchymal stromal cells after 3 days of dynamic culture in the microfluidic device. This proof-of-principle study, therefore, marks a significant step forward in manufacturing submicron structure-on-a-chip models for bone regeneration studies.

KEYWORDS: submicron pillars, two-photon polymerization, microfluidics, bone regeneration

\section{INTRODUCTION}

Bone regeneration is a complex biological process that supports skeletal health throughout our lifetime. In this process, osteoprogenitor cells migrate, proliferate, and differentiate to restore and repair bone injuries. ${ }^{1,2}$ However, the bone regeneration capacity of the human body is either inadequate or is compromised in the case of large bony defects, infections, arthritis, and tumors. ${ }^{3-6}$ The innate bone regeneration capacity should therefore be augmented through biomaterials that promote osteogenesis, thereby enhancing the performance of the bone regeneration process. ${ }^{6-8}$ The vast majority of such biomaterials introduced to date work on the basis of locally delivering biomolecules such as bone morphogenic protein (BMP) to promote the osteogenic differentiation of human mesenchymal stromal cells (hMSCs). 9,10 There are, however, concerns regarding the dose-dependent toxicity effects of some of these biomolecules $^{10-12}$ particularly in larger animals such as humans. In addition, such biomolecules are often expensive and may have undesired side effects. ${ }^{12,13}$

An alternative approach tries to exploit the effects of (submicron/nanoscale) topographies on cellular processes including migration, adhesion, proliferation, and differentiation to enhance the bone tissue regeneration performance of biomaterials. ${ }^{14,15}$ In particular, several studies have shown that very specific shapes and dimensions of nanopatterns could stimulate the osteogenic differentiation of stem cells. ${ }^{16-18}$ Moreover, the same nanoscale patterns could be used to kill bacteria so as to minimize the risk of implant-associated infections. ${ }^{19-21}$ However, it is still unclear how different design parameters of the surface nanopatterns affect the various cellular processes both for the host cells and bacteria. ${ }^{22,23}$ One

Received: July 29, 2019

Accepted: September 27, 2019

Published: September 27, 2019 
(a)

Two-photon polymerization Dill configuration

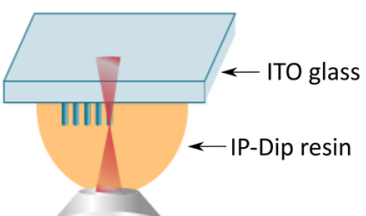

$\longleftarrow 63 x$-Objective lens

Upscaling patterns

$\downarrow$

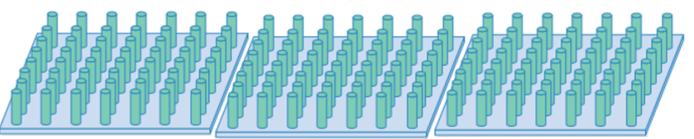

Bonding through Oxygen plasma

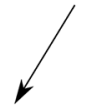

(b)

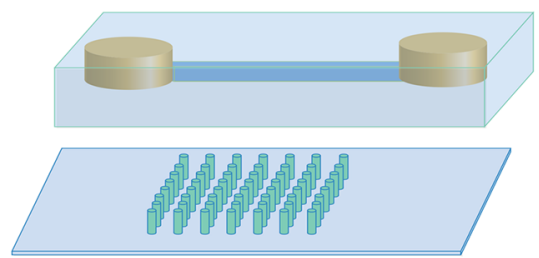

(d)

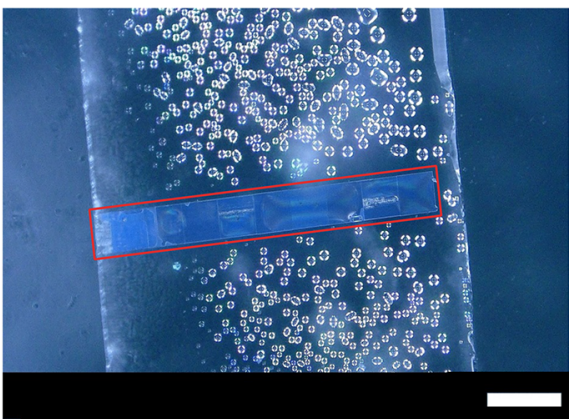

(c)
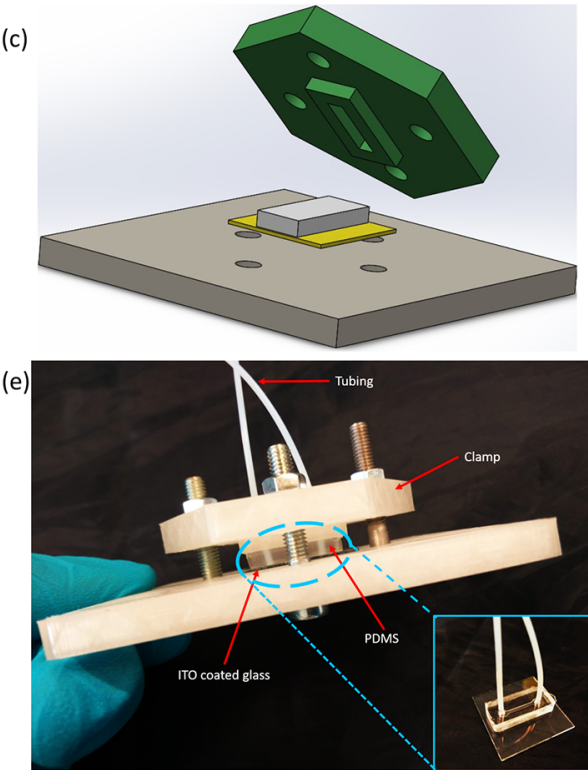

Figure 1. (a) Schematic illustration of the 2PP process to prototype a large area of submicron patterns. (b) Oxygen plasma bonding process for cell study. ( $\mathrm{c}$ and e) SolidWorks and real view models of the mechanical clamp setup used to align the channel over the topography in the submicron pattern delamination test. (d) Aligned submicron patterns across the channel width. Microdroplets can be seen after the delamination experiment. Scale bar is $200 \mu \mathrm{m}$.

therefore has to perform many experiments to systematically study the effects of a large number of design parameters on the different types of cellular responses. This challenge is further compounded by the fact that the experiments have to be repeated for different cell types including stem cells, immune cells, and different types of bacteria (e.g., Gram-positive and Gram-negative). Therefore, high-throughput experimental setups that allow for such large systematic studies are required. Lab-on-a-chip devices that incorporate various submicron to nanoscale patterns inside a microfluidic chip are very attractive solutions for such an application. Such devices also allow improving the mass transfer of nutrients, metabolites, gases, and metabolic byproducts while controlling the temperature and $\mathrm{pH}^{24}$ Moreover, these in vitro models may provide an alternative for the in vivo models that involve animal testing. However, fabrication of such devices would require a multiscale manufacturing approach to create the patterned surfaces and to integrate them into the chip.

Micro- and nanoscale patterns can be fabricated by removing material from a bulk material using top-down nanopatterning techniques such as lithography ${ }^{25}$ and etching. ${ }^{26,27}$ These structures can be also manufactured by deposition of atoms, molecules, or nanoparticles through bottom-up nanopatterning (e.g., focused electron beam induced deposition (FEBID), ${ }^{28}$ chemical vapor deposition $(\mathrm{CVD}),{ }^{29}$ and molecular self-assembly ${ }^{30}$ ). Although some of these techniques are capable of producing features with ultrafine resolutions $(\leq 100 \mathrm{~nm})$, they are often restricted in terms of surface quality, upscaling, and combination and alignment of multiple elements. To overcome these restrictions, here, we used three-dimensional (3D) direct laser writing (DLW). ${ }^{31,32}$ DLW works on the basis of two (or 
multiple) photons polymerization (2PP) and allows for printing $3 \mathrm{D}$ objects at the nanoscale and larger (up to $\mathrm{mm}$ ) feature sizes. ${ }^{33}$ Barata et al. ${ }^{33}$ developed a microfluidic chip integrated with microstructures through a combination of photolithography, 2PP, and hot embossing. They studied the interaction of human osteosarcoma cells with the biomaterial under a low flow rate of continuous medium perfusion. In another study, Yong et al. ${ }^{34}$ developed a microfluidic device incorporated with nanogratings through a stitching and microtransfer assembly technique rooted in polymer thin film technologies. Despite the availability of these few studies, a versatile technique to fabricate microfluidic devices endowed with submicron/nanoscale features is still lacking. ${ }^{24}$

Manufacturing a large area of submicron/nanopatterns for cell culture and preserving the geometrical accuracy after assembly are the two main challenges in the integration of nanopatterns within microfluidic devices. ${ }^{24}$ In this study, we fabricated large patterned areas with submicron pillars by using the $2 \mathrm{PP}$ process and embedded them in a microfluidic chip (Figure $1 \mathrm{a}-\mathrm{d}$ ). The uniformity of the submicron pillars was boosted by proper selection of the materials and writing strategies, as well as by correcting for the substrate tilt. The morphologies of the pillars were first characterized and then upscaled to a larger area ( $\mathrm{mm}$ range). We then fabricated a microscaffold covered with submicron pillars of various heights. In addition to measuring the adhesion force and wettability of the submicron pillars, we performed dynamic culture of hMSCs to evaluate the cytocompatibility of the patterned surfaces.

\section{MATERIALS AND METHODS}

2.1. Submicron Patterning Using 2PP. We used the 2PP process for additive manufacturing of the surface submicron patterns using a Photonic Professional GT (Nanoscribe, Germany) machine. This machine works on the basis of a pulsed femtosecond laser (center wavelength $=780 \mathrm{~nm}$, pulse duration $=100 \mathrm{fs}$, repetition rate $=80 \mathrm{MHz}$ ) and is equipped with a $63 \times$ objective lens (NA 1.4, numerical aperture). The Piezo writing strategy and dip-in laser lithography (DiLL) configuration were selected for writing the submicron-scale features. The submicron pillars were created using an acrylate-based resin (IP-Dip, Nanoscribe, Germany) atop an indiumtin oxide (ITO) coated fused silica glass (ITO glass) (Nanoscribe, Germany). Before printing, the substrates were cleaned with acetone and isopropyl alcohol (IPA; both Sigma-Aldrich, Germany). After writing, the samples were developed in propylene glycol monomethyl ether acetate (PGMEA, Sigma-Aldrich, Germany) for $25 \mathrm{~min}$, followed by $5 \mathrm{~min}$ of development and rinsing in IPA.

2.2. Upscaling of Submicron Patterns and Fabrication of a Multiscale 3D Scaffold. The writing parameters of the $2 \mathrm{PP}$ process were first optimized for printing reproducible submicron pillars of known dimensions in an area of $100 \mu \mathrm{m} \times 100 \mu \mathrm{m}$. Larger areas of this nanotopography (up to $2 \mathrm{~mm} \times 1.7 \mathrm{~mm}$ ) were then achieved by repeat-printing the previously optimized area. The procedure was then extended to submicron pillars with various heights on a $3 \mathrm{D}$ woodpile structure, which was fabricated using the machine's Galvo strategy which has a larger throughput. Each rod of the woodpile had a cross section of $5 \mu \mathrm{m} \times 5 \mu \mathrm{m}$ and a length of $55 \mu \mathrm{m}$, and the rods were separated by $10 \mu \mathrm{m}$. Two layers were printed and the overall footprint of the woodpile was $55 \mu \mathrm{m} \times 55 \mu \mathrm{m}$.

2.3. Characterization of the Submicron Patterns. Submicron topographical surfaces were characterized using a scanning electron microscope (Jeol InTouchScope JSM-6010LA, Japan). All specimens were sputter-coated with a $\sim 10 \mathrm{~nm}$ thick gold-palladium layer before imaging. Furthermore, an optical microscope (Keyence Digital Microscope VHX-6000, U.S.A.) was used to acquire pixel intensity maps and to evaluate the reproducibility and uniformity of the submicron pillar dimensions. The wettability of the nonpatterned, patterned, and flat polymer surfaces was assessed using a drop shape analyzer (KRUSS DSA100, Germany). First, the samples were cleaned with IPA (Sigma-Aldrich, Germany). Then, droplets of deionized water $(0.1-0.3 \mu \mathrm{L})$ were laid on the nanopatterned and nonpatterned surfaces. The contact angles were recorded after $5 \mathrm{~s}$ (three measurements per sample).

2.4. Image Processing and Quantification. The acquired SEM images were analyzed using ImageJ (open source image analysis software, http://rsb.info.nih.gov/ij/index.html) to measure the height, diameter, and uniformity of the patterns over a large area. To do so, the height and diameter of the submicron pillars were first quantified through high-magnification SEM images at $13000 \times$ from a few sample regions within the patterned area that were then used to calibrate the mesoscale image of the submicron patterns obtained from low-magnification images (taken at 850X via SEM and stitched together). Furthermore, optical images of the same area were taken to analyze their pixel intensity map. Finally, the pixel intensity map of the optical images was correlated with the data obtained from highmagnification SEM images using a custom-made MATLAB code $(2017 b)$ to identify the numbers, dimensions (mean $\pm S D$ ), and uniformity of the printed submicron pillars (Table 2).

2.5. Fabrication of the Microfluidic Device. A single microchannel of $200 \mu \mathrm{m}$ height, $1 \mathrm{~mm}$ width, and $7.85 \mathrm{~mm}$ length was fabricated via the mold replication technique. First, the mold was 3D printed using the stereolithography technique (Envisiontec Micro Plus HD, Germany). Then, a mixture of polydimethylsiloxane (PDMS) (Sigma-Aldrich, Germany) and the curing agent (10:1 w/ w) (Sigma-Aldrich, Germany) was poured into the 3D printed molds and desiccated for $1 \mathrm{~h}$ to remove any trapped air bubbles. After $3 \mathrm{~h}$ curing at $70^{\circ} \mathrm{C}$, the PDMS part was peeled off the molds. The PDMS layer was then placed over the substrate while aligning the channel with the patterns (Figure $1 \mathrm{~b}, \mathrm{~d}$ ). The method of bonding PDMS to the substrate depended on the type of the experiment performed later. For the delamination test, PDMS was pressed onto the substrate through a mechanically designed clamp, while oxygen plasma was used in the case of the specimens prepared for cell culture.

2.6. Delamination Test. The delamination of the submicron pillars was tested with respect to the various flow rates of deionized water. Patterned strips of $1 \mathrm{~mm} \times 0.1 \mathrm{~mm}$ were integrated into the microfluidic chip and were held leak-tight with the help of the clamp. The computer-aided design (CAD) model and the actual 3D printed mechanical clamp (made with an Original Prusa I3MK2S printer, Czech Republic) holding the chip are presented in Figure 1c,e, respectively. The different flow rates of deionized water $(1,3,5$, and 8 $\mathrm{mL} / \mathrm{min}$ ) were applied in the chip through a peristaltic pump (Ismatec ISM915, Germany). The submicron pillars were observed before and after the experiments using optical microscope and SEM to check whether they were delaminated.

2.7. Dynamic Cell Culture. Two $1 \mathrm{~mm} \times 0.3 \mathrm{~mm}$ areas of patterned and nonpatterned sheets (with a height of $3 \mu \mathrm{m}$ ) were printed in parallel and aligned along the channel edge. Both the patterned surface and the channel side of the PDMS were exposed to air plasma (Low Cost System Atto, Diener Electronic, Germany) for 2 min (power level $=40 \mathrm{~W}$, chamber pressure $=4 \mathrm{mbar}$ ) after which they were brought into contact.

hMCSs (passage 7; Lonza, Switzerland) were cultured in alpha minimal essential medium ( $\alpha$ MEM: $10 \%(\mathrm{v} / \mathrm{v})$ fetal calf serum (FCS), pH 7.5, phenol-red free; Life Technologies, U.S.A.) as previously described in detail by us. ${ }^{35}$ A cell seeding concentration of approximately $1.1 \times 10^{6}$ cells $/ \mathrm{ml}$ was used for the dynamic cell culture. The microfluidic device was flushed 3 times with $\alpha \mathrm{MEM}$ medium followed by seeding into the microchannel. The cells were then incubated (at $37^{\circ} \mathrm{C}, 5 \% \mathrm{CO}_{2}$ ) for $6 \mathrm{~h}$ before starting the flow of culture medium at $10 \mu \mathrm{L} / \mathrm{h}$, using a NE-1200 syringe pump (KF Technology, Italy). After 3 days, the cells were rinsed 2 times with phosphate buffered saline (PBS, Life Technologies, U.S.A.) and incubated $10 \mathrm{~min}$ in a fixative solution (4\% paraformaldehyde (PFA) and $1 \%$ glutaraldehyde (GA) (Sigma-Aldrich, Germany) in PBS) at room temperature. The fixed samples were washed 2 times with 
distilled water for $5 \mathrm{~min}$ and then immersed in a series of graded alcohol-PBS solution (50\%, 70\%, and $96 \%$ ) for 15,20 , and $20 \mathrm{~min}$, respectively. The samples were dried overnight at room temperature. At last, the specimens were gold-sputtered before obtaining SEM images.

2.8. Cell Viability. A live/dead cell assay (ThermoFisher Scientific, U.S.A.) was performed to study the viability of the cultured cells. After 3 days of dynamic cell culture, hMSCs were washed 3 times with PBS and incubated in a mixture of $5 \mu \mathrm{g} / \mathrm{mL}$ Hoechst 33342 (Nuclei Dye, Sigma-Aldrich, Germany) and $10 \mathrm{mg} / \mathrm{mL}$ propidium iodide (Dead Dye, Sigma-Aldrich, Germany) for $15 \mathrm{~min}$ at $37{ }^{\circ} \mathrm{C}$. In this fluorescence-based assay, Nuclei Dye stains the nucleus of both live and dead cells in blue while Dead Dye penetrates the membrane of the dead cells (red). After staining, the cells were washed with PBS and viewed with a confocal microscope (Axiovert 200MOT, Zeiss, Germany).

\section{RESULTS AND DISCUSSION}

3.1. Fabrication of Reproducible Uniform Submicron Patterns. Selection of an appropriate print configuration and writing parameters for the $2 \mathrm{PP}$ process is necessary to produce uniform and reproducible submicron patterns. To further enhance the uniformity of the patterns, a tilt correction strategy for substrate was adopted and the effects of different configurations were evaluated. Lastly, a parametric study was conducted to assess the effects of $2 \mathrm{PP}$ writing parameters on the uniformity of the submicron patterns and the minimum feature size that could be reliably achieved.

3.1.1. Effects of Print Configurations. Different configurations and respective materials (resins and substrates) for the $2 \mathrm{PP}$ process were evaluated (Table 1 ). In comparison with the

Table 1. Configurations, Materials, and the Interface Signal Amplitude in the 2PP Process

\begin{tabular}{llc} 
configuration & \multicolumn{1}{c}{ materials } & $\begin{array}{c}\text { interface signal } \\
\text { amplitude }\end{array}$ \\
DiLL & $\begin{array}{c}\text { IP-Dip + ITO coated fused silica } \\
\text { glass }\end{array}$ & $1000-2000$ \\
DiLL & IP-Dip + fused silica glass & $600-700$ \\
conventional & IP-L 780 + borosilicate glass & $300-400$ \\
\hline
\end{tabular}

other types of substrates, a significant improvement (up to $86 \%$ ) in the uniformity of the submicron patterns was achieved by printing over the ITO-coated fused silica glass when scaled up to a $2 \mathrm{~mm} \times 1.7 \mathrm{~mm}$ area in the DiLL configuration (Figure 2a). The ITO-coated fused silica substrate in combination with IP-Dip resin yielded a higher interface signal amplitude (a value directly proportional to the refractive index mismatch at the interface of the resin and the substrate) in comparison with an uncoated fused silica substrate (Figure S1, Supporting Information). When using the conventional configuration (IPL 780, borosilicate glass), the signal amplitude decreased even further (Figure S2, Supporting Information). Enhancing the interface signal amplitude decreased the noise and helped in more accurate detection of the substrate surface. This noise was decreased by increasing the refractive index difference between the two media (Figures S1 and S2, Supporting Information).

3.1.2. Effects of Tilt Correction. The Piezo writing strategy was selected among the available writing strategies in the $2 \mathrm{PP}$ process to produce each submicron pillar as a single voxel. At the submicron scale, a large variation in the pillar dimensions along the direction of writing was observed due to the angular motion of the scanner during the writing process (Figure S3a,
Supporting Information). We used a tilt compensation method to reduce such effects where a correction accounting for the tilt of the substrate (accuracy in the range of $0.01^{\circ}$ ) was made to obtain a more uniform pattern (Figure S3c, Supporting Information). This procedure required measuring the interface position (in the $z$-direction) followed by fitting a linear plane to the measured position data points. The tilt angle of this fitted plane in the $x$ - and $y$-direction were then input into the 2PP machine program to add the appropriate offsets in the $z$ coordinate while traversing in the $x$ - and $y$-directions. The uniformity of the fabricated submicron pillars increased after applying the tilt correction to the ITO-coated sample (Figure $2 \mathrm{~b})$. The uniformity of the patterns written on manually tiltcorrected, auto tilt-corrected, and uncorrected substrates (ITO fused silica, DiLL configuration) are compared in the supplementary document (Figure S3).

3.1.3. Effects of 2PP Writing Parameters. Achieving submicron/nano patterns of specific dimensions involves optimizing a number of parameters such as the laser power, time of exposure, interface distance, and other Piezo writingbased parameters. Submicron pillars as small as $160 \mathrm{~nm}$ in diameter and $300 \mathrm{~nm}$ in height could be fabricated using the Piezo writing strategy (Figures S4, Supporting Information). The height and diameter of the submicron pillars changed together with the laser parameters. It is therefore challenging to fix one of these dimensions, while varying the other. The higher values of the laser power and exposure time caused the diameters of the submicron pillars to increase (Figures S4, Supporting Information), while the height and diameters of the submicron pillars decreased with the interface distance (Figures S5, Supporting Information). The adhesion of the submicron pillars to the substrate was also decreased by the interface distance (Figures S6, Supporting Information). Increasing the interface distance, however, caused the printing process to start deeper into the substrate and reduced the uniformity and reproducibility of the submicron pillars (Figure S6, Supporting Information). The uniformity of the submicron pillars improved with the laser power and exposure time (Figure S7, Supporting Information). After optimization of all these $2 \mathrm{PP}$ parameters, a laser power of $27.2 \mathrm{~mW}$, an exposure time of $2400 \mu \mathrm{s}$, and an interface distance of $0.3 \mu \mathrm{m}$ were selected to achieve reproducible and uniform patterns (Figure $2 \mathrm{c}$ ). The ellipsoidal shape of the submicron pillars (Figure 2c) were similar to that of the focal point of a focused laser beam. The focal point shape originates from the nature of the diffraction and cannot be improved by lens design or through adjustment of the optical system. ${ }^{36}$ However, it is possible to adjust the shape of voxel using such methods as shaded ring filters, equivalent phase and amplitude masks, or even approaches inspired by the stimulated emission depletion (STED) technique. ${ }^{37-39}$

3.2. Characterization of Submicron Patterns. 3.2.1. Measuring Uniformity over a Large Area. An area of $1 \mathrm{~mm} \times 0.1 \mathrm{~mm}$ was patterned using the above-mentioned optimal laser parameters (Figure 2d, e). The uniformity of this printed topography can be quantified through the pixel threshold intensity values. The pixel threshold intensity can be detected by correlating the histogram describing the distribution of the submicron pillar diameters (Figure 2f) with the data regarding the distribution of the pixel intensity (Figure $2 \mathrm{~g}$ ). Here, the uniformity of the patterns is defined as the percentage of the area in which the diameter of the submicron pillars is larger than the cutoff value (i.e., submicron 
(a)

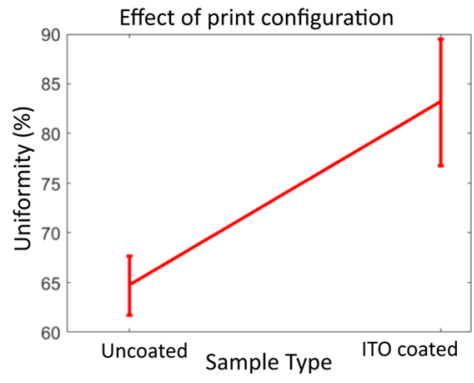

(b)

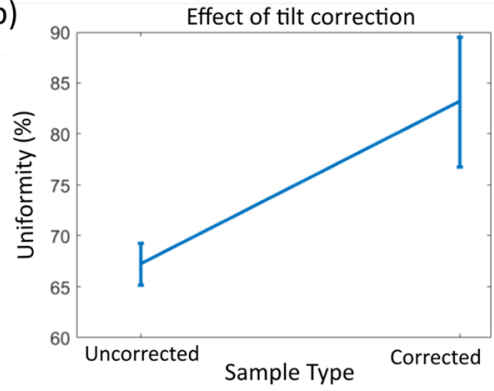

(c)

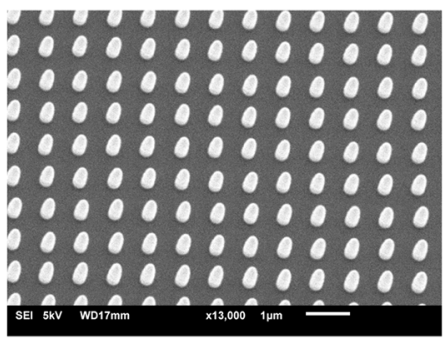

(d)

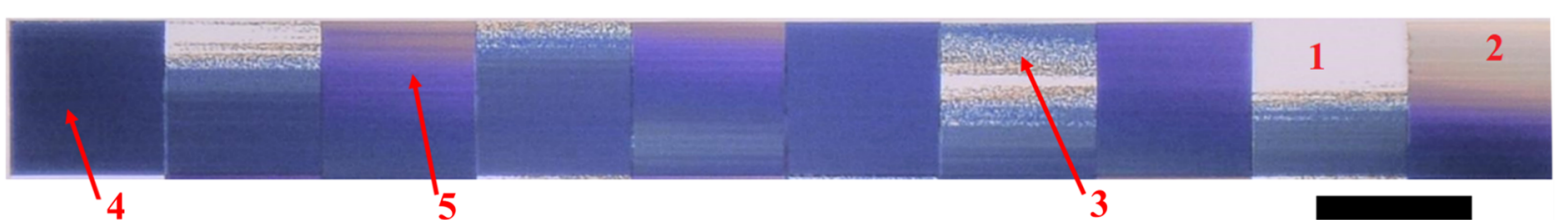

(e)
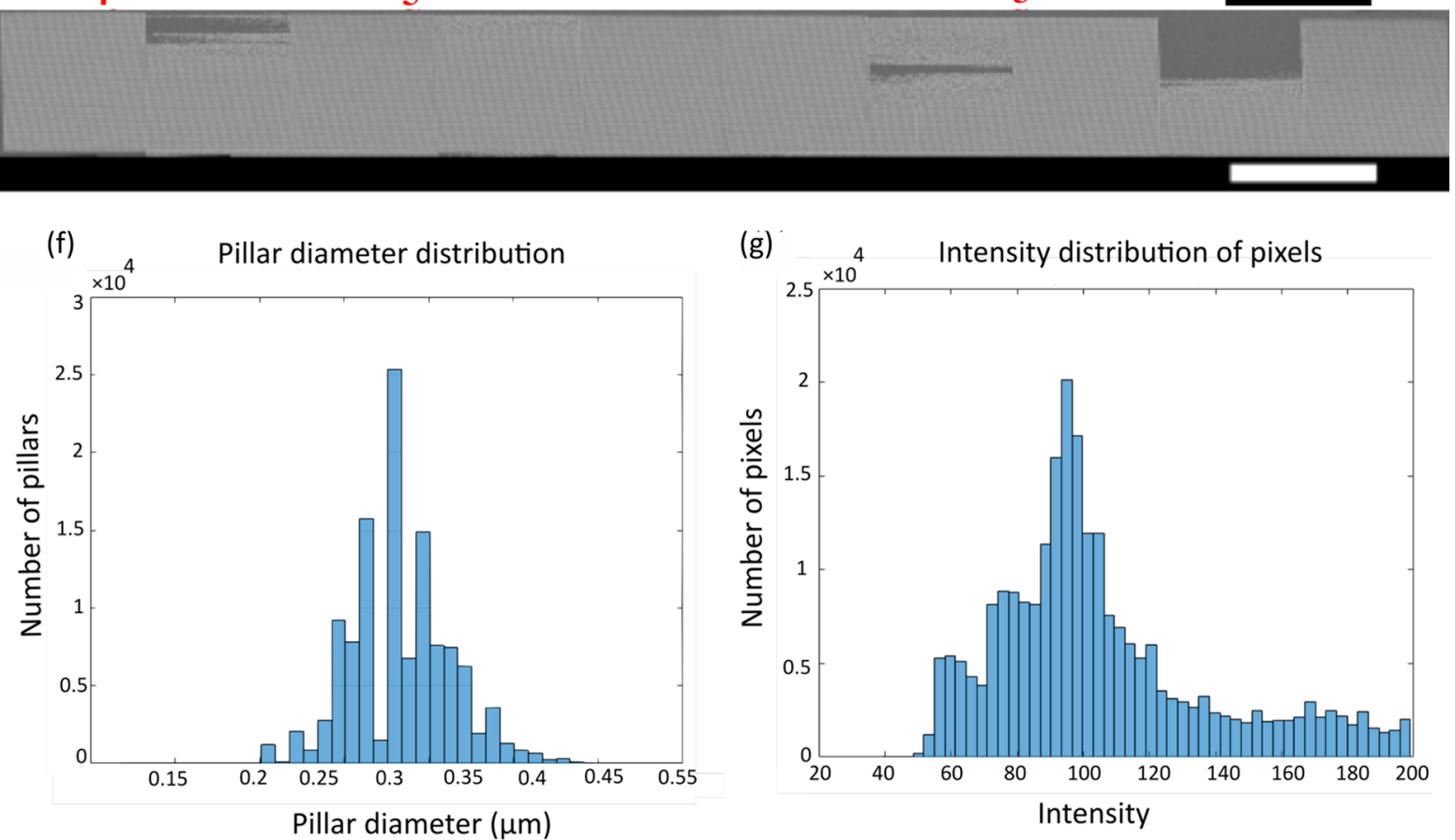

(h)

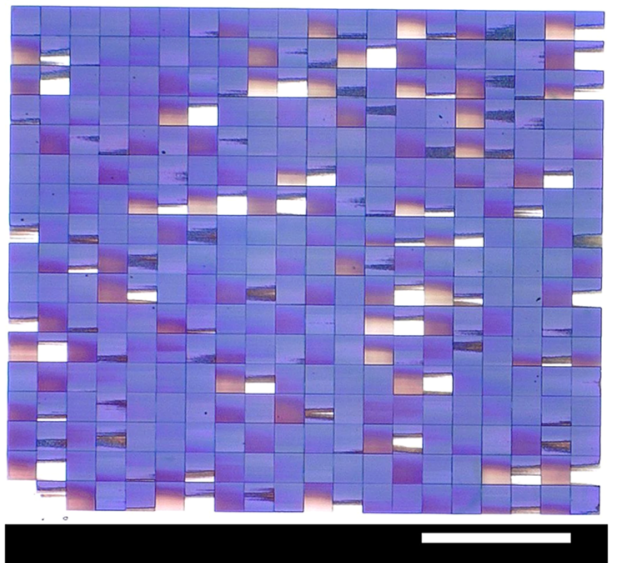

(i)

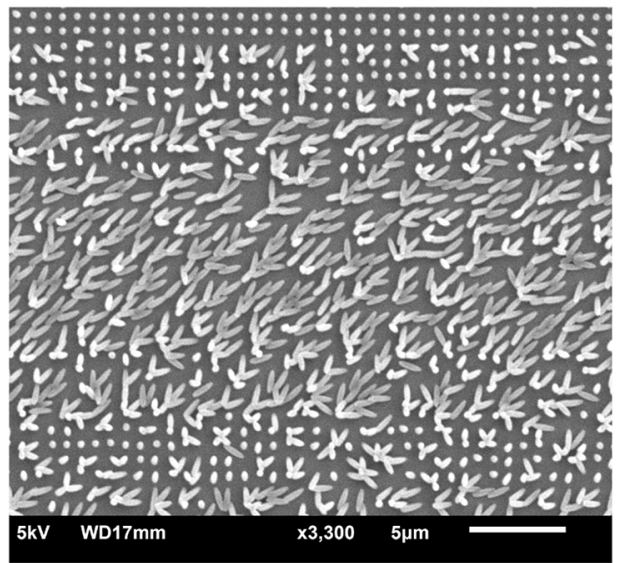

Figure 2. ( $\mathrm{a}$ and $\mathrm{b}$ ) Effects of the print configuration and tilt compensation on the uniformity of the fabricated submicron pattern $(n=4)$. Both cases (a) and (b) were compensated for tilts. (c) Morphological characterization of uniform submicron pillars (the scale bar is $1 \mu \mathrm{m}$ while the tilt angle is $20^{\circ}$ ). (d) Optical image of the area depicting the different regions covered by submicron patterns (the scale bar is $100 \mu \mathrm{m}$ ). (e) Stitching low-resolution SEM images to create an area of $1 \mathrm{~mm} \times 100 \mu \mathrm{m}$ (the scale bar is $100 \mu \mathrm{m}$ ). (f) The frequency distribution of the diameters of the submicron pillars determined using SEM image (e). (g) The frequency distribution of the pixel intensities obtained from the optical image (d). (h) The patterned surface was extended to a $2 \mathrm{~mm} \times 1.7 \mathrm{~mm}$ area (the scale bar is $500 \mu \mathrm{m}$ ). (i) Morphological characterization of the submicron pillars from the damaged area (region 3 in d). The scale bar is $5 \mu \mathrm{m}$. 
pillar diameter $\geq$ (mean diameter $-50 \mathrm{~nm}))$. An upper limit of about $500 \mathrm{~nm}$ was also applied on the diameter to avoid counting delaminated submicron pillars stuck to the substrate in this analysis. Through this method, about $73 \%$ of the area (Figure 2d) has pillar diameters higher than $284 \mathrm{~nm}$ (uniformity is calculated using the data presented in Table 2 ). These submicron patterns upscaled to an area of $2 \mathrm{~mm} \times$

Table 2. Specifications of the Submicron Pillars (Obtained from Image Analysis)

$\begin{array}{ll}\text { expected number of submicron pillars } & 156250 \\ \text { actual number of submicron pillars } & 118966 \\ \text { interspace (center to center) } & 800 \mathrm{~nm} \\ \text { mean diameter }(d) \pm S D & 334 \pm 30 \mathrm{~nm} \\ \text { mean height }(h) \pm S D & 1541 \pm 60 \mathrm{~nm}\end{array}$

$1.7 \mathrm{~mm}$ (Figure $2 \mathrm{~h}$ ) with a printing time of about $87 \mathrm{~h}$ and 50 min. This process is therefore not an ideal option for mass production of submicron patterned areas but rather for the rapid submicron patterning of large areas. The uniformity of this area was measured to be about $86 \%(n=10)$.

Several regions could be seen after printing over larger areas (Figure 2d). Region 4 showed a uniform distribution of submicron pillars, while the color intensity decreased in region 5 , indicating a decrease in the pillar diameter. A further drop in the pillar diameter created areas similar to the region 2 . There were also areas where there are no submicron pillars (region 1) or areas where submicron pillars were collapsed (Figure $2 \mathrm{i}$ and region 3 in Figure 2d) during the development process in PGMEA and IPA. One reason could be the weak adhesion of the submicron pillars to the substrate. This usually happens when the laser beam focuses far above the substrate surface or when the edge of the voxel that is going to be printed is too close to the surface of the substrate. ${ }^{36}$ Collapse of submicron pillars could also happen due to the capillary forces during sample drying. Nano/micro pillars with high aspect ratios and dense arrays are more prone to deformation by capillary forces. ${ }^{40}$ A postprocessing step such as $\mathrm{CO}_{2}$ critical point drying can help in enhancing the uniformity of the submicron pillars. ${ }^{41,42}$ Although printing over large areas could not give us $100 \%$ uniform submicron patterns in this study, the uniformity significantly enhanced after optimization of $2 \mathrm{PP}$ parameters in the Piezo writing strategy, testing various print configurations, and applying a manual tilt correction.

3.2.2. Wettability. Water contact angles on the nonpatterned glass (ITO-glass), patterned glass, and flat polymeric surface were $81.84 \pm 3.15^{\circ}, 41.7 \pm 3.9^{\circ}, 73 \pm 3.5^{\circ}(n=9)$, respectively (Figure 3a). Previous studies ${ }^{43-45}$ reported a water contact angle of $60^{\circ}-80^{\circ}$ for the ITO glass. This range indicates that the angle depends on the precleaning procedure and may change due to the different solvent cleaning processes and plasma treatments. ${ }^{46}$ In this study, exposure to chemicals such as acetone, IPA, and PGMEA had been considered due to the involved sample development process. The Wenzel's model $^{47}$ (eq 1), describing a homogeneous wetting regime, was used to estimate the contact angle of uniform patterns wetted by droplets.

$$
\cos \theta_{\mathrm{w}}=R \cos \theta_{\mathrm{y}}
$$

Here, the apparent contact angle of the droplet on a patterned and a smooth nonpatterned surface is given by $\theta_{\mathrm{w}}$ and $\theta_{\mathrm{y}}$, respectively. ${ }^{47,48}$ On a rough surface, the roughness factor is determined by $R$ which describes the ratio of the actual surface area to the apparent surface area of a rough surface. In this
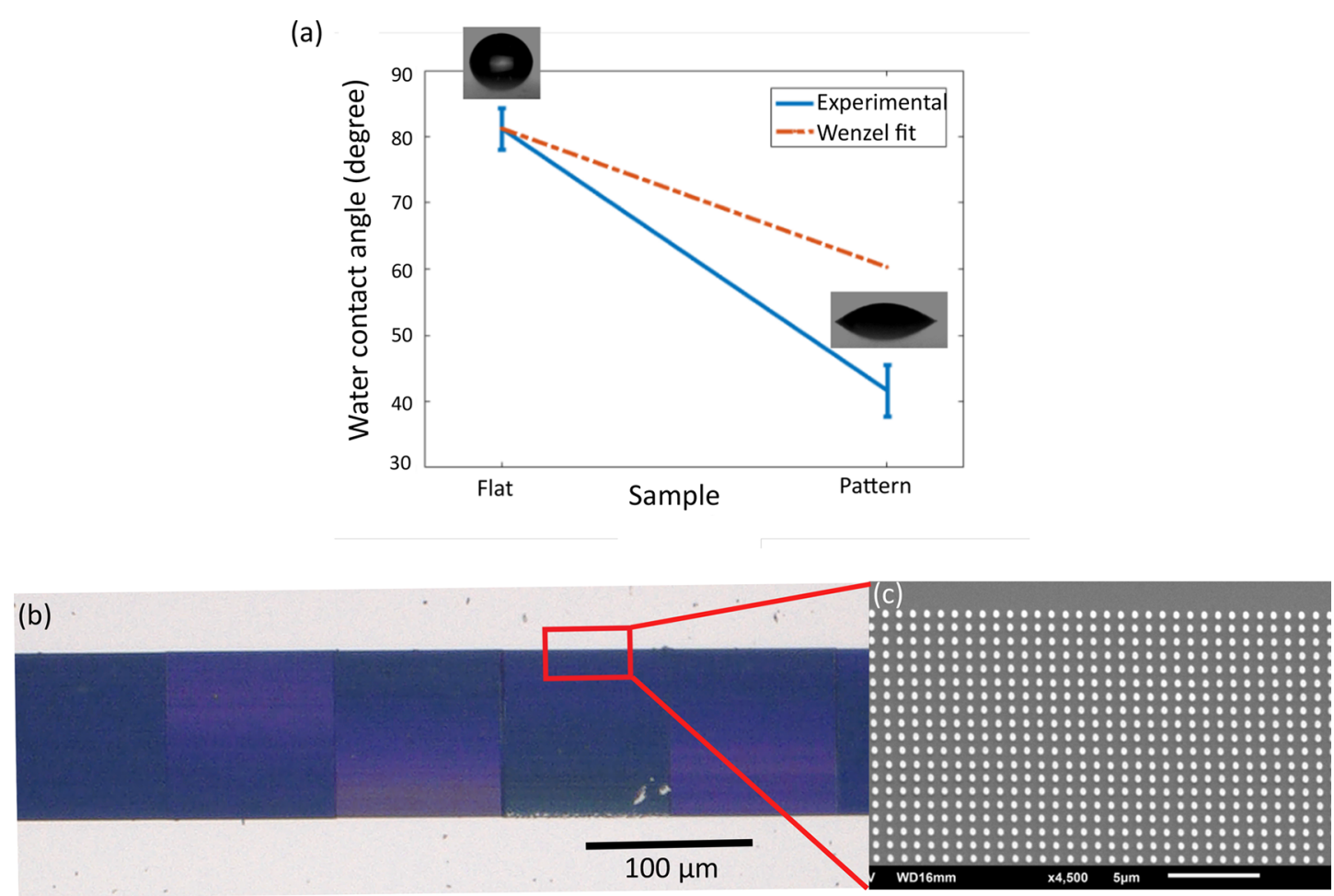

Figure 3. (a) Water contact angle measurements of the flat and patterned areas. (b and c) The optical and SEM images of the submicron pillars after performing the microfluidic delamination tests at a flow rate of $8 \mathrm{~mL} / \mathrm{min}$. No signs of delamination were observed. The scale bar is $100 \mu \mathrm{m}$ in (b) and $5 \mu \mathrm{m}$ in (c). The tilt angle is $20^{\circ}$ in (c). 
study, by assuming cylindrical submicron pillars of height $h$, diameter $d$, and pitch $p$, the roughness factor can be calculated using eq 2 as ${ }^{47}$

$$
R=1+\frac{\pi d h}{p^{2}}
$$

By substitution of the dimensions (Table 2 ) in this equation, the roughness factor is calculated to be $R \approx 3.49$. Replacement of this value into eq 1 yields $\theta_{\mathrm{w}}=60.3^{\circ}$, which is higher than the experimental value of the contact angle $\left(47.1^{\circ}\right)$. The deviation seen in the experimental value of the contact angle from the one predicted by the Wenzel's model could be attributed to the small nonhomogeneity in patterns (due to process limitation, there are few regions with imperfect submicron pillars) and assuming submicron pillars as cylindrical rather than using their actual truncated ellipsoid shape. This model, however, does correctly predict the increase in the hydrophilicity in such a case.

3.2.3. Adhesion Force of the Submicron Pillars. The shear stress on the microchannels walls of $7.85 \mathrm{~mm}$ length $(l), 1 \mathrm{~mm}$ width $(w), 200 \mu \mathrm{m}$ height $(H)$, and a flow rate $Q$ (liquid: water with a dynamic viscosity $\left.=8.9 \times 10^{-4} \mathrm{~Pa} \mathrm{~s}\right)$ was measured through eq $3^{49-51}$ (Table 3$)$.

$$
\tau=6 \mu Q / w H^{2}
$$

Table 3. Microfluidic Testing Conditions Used to Test the Resistance of the Submicron Pillars against Delamination

\begin{tabular}{ccc}
$\begin{array}{c}\text { flow rate } \\
(\mathrm{mL} / \mathrm{min})\end{array}$ & $\begin{array}{c}\text { shear stress } \\
(\mathrm{Pa})\end{array}$ & \multicolumn{1}{c}{ comments } \\
1 & 2.2 & $\begin{array}{l}\text { submicron pillars unaffected, no leakage } \\
\text { submicron pillars unaffected, no leakage }\end{array}$ \\
3 & 6.7 & $\begin{array}{c}\text { submicron pillars unaffected, occasional } \\
\text { leakage } \\
5\end{array}$ \\
8 & 11.2 & $\begin{array}{c}\text { submicron pillars unaffected, leakage via } \\
\text { inlet/outlet }\end{array}$ \\
\hline
\end{tabular}

Leakage was occasionally observed for a flow rate of $5 \mathrm{~mL}$ / min. Above this flow rate, keeping the chip leak tight was impossible (especially at the inlet and outlet connection points). The submicron pillars, on the other hand, remained unaffected by flow rates of up to $8 \mathrm{~mL} / \mathrm{min}$. Figure $3 \mathrm{~b}, \mathrm{c}$ show pictures of pillars that had been exposed to such flow rates. It can therefore be concluded that the wall shear stress should be more than a few tens of Pascal to delaminate the submicron pillars.

The following equation gives the shear force applied to the pillars during the microfluidic flow studies:

$$
\begin{aligned}
d F_{\text {drag }} & =\frac{1}{2} \rho C_{\mathrm{D}} 2 r \int_{0}^{h} v^{2}(z) \mathrm{d} z \\
F_{\text {drag,tot }} & =\rho C_{\mathrm{D}^{r}} r \int_{0}^{h} v^{2}(z) \mathrm{d} z \\
& =\rho C_{\mathrm{D}} r \int_{0}^{h} v_{\max }^{2}\left(1-\frac{\left(R_{\mathrm{h}}-z\right)^{2}}{R_{\mathrm{h}}^{2}}\right)^{2} \mathrm{~d} z
\end{aligned}
$$

where $v(z)$ is the flow velocity inside the channel, $r$ is the pillar radius, $R_{\mathrm{h}}$ is the hydraulic radius of the micro channel, and $h$ is the height of the pillars. As the flow regime inside the channel is laminar (at the maximum flow rate: $8 \mathrm{~mL} / \mathrm{min}$, Reynolds number $=284)$, the drag coefficient leads to 1 at this Reynolds number. ${ }^{52}$ By substituting $h=1.5 \mu \mathrm{m}$ and $r=167 \mathrm{~nm}$ in the eq $4, F_{\text {drag,tot }}$ is estimated to be of the order $10^{-15} \mathrm{~N}$ and could, therefore, be neglected (section S4, Supporting Information).

Submicron pillars were also experiencing a traction force by hMSCs cultured on them, which could lead to bending and delaminating the pillars (Figure 5e). The bending force on the pillars can be estimated using the following equation: ${ }^{53}$

$$
\delta=4 F h^{3} / 3 \pi E r^{4}
$$

where $\delta$ is the linear horizontal displacement of the pillar tips, $F$ is the horizontal bending force, $h$ is the height of the pillar, $r$ is the radius of the pillar, and $E$ is the Young's modulus of the polymeric material. $\delta$ can be estimated to be in the range of a few hundred nanometers from the SEM images (Figure $5 \mathrm{e}$ ), $E$ to be in the range of a few $\mathrm{GPa}^{54} r \approx 167 \mathrm{~nm}$, and $h \approx 1.54$ $\mu \mathrm{m}$. These estimations resulted in calculated forces in the order of hundreds of $\mathrm{nN}$ to a few $\mu \mathrm{N}$. This estimated range of forces is in agreement with the values reported by $\mathrm{Fu}$ et al. ${ }^{55}$

The forces applied by the cells to the submicron pillars are much higher than the drag forces. One could therefore conclude that the cell traction forces delaminated the submicron pillars during the dynamic cell culture experiments.

3.3. Fabrication of Multiscale Structures. The possibility of printing 3D micro architectures such as porous scaffolds with controlled porosities and nanotopographies using the 2PP process enables new approaches to the design of biomaterials. Cha et al. ${ }^{56}$ studied the effects of micropatterns added on microscaffolds on the cellular behavior of preosteoblast cells. They investigated two types of patterns (i.e., micropillars and microridges). The minimum feature size of the patterns was $2 \mu \mathrm{m}$. By comparison, the incorporation of submicron/nanoscale patterns on the surface of microscaffolds through the application of the $2 \mathrm{PP}$ technique is much more difficult while highly effective in inducing unique cellular responses. ${ }^{17}$ In this study, we showed the effects of different 2PP parameters on the characteristics of the submicron patterns. Based on these findings, we succeeded to print submicron pillars with diameters down to $200 \mathrm{~nm}$ with great precision not only on large areas of flat surfaces but also on the surface of 3D microstructures (Figure 4a). In addition, we reproduced submicron pillars with height gradient on the surface of our microscaffolds (Figure $4 b$ and $c$ ). The potential of shifting between the Galvo and Piezo writing strategies in a single process allowed for this novelty. The Galvo writing strategy was used to fabricate the 3D woodpile structure, while the Piezo writing strategy was used to incorporate the submicron patterns on the woodpile layers. It is also worth mentioning that increasing the distance from the woodpiles surface leads to a gradient in the height and diameter of the submicron pillars created on the surface of various woodpiles. This approach could be used to create 3D cellular microenvironments that resemble more closely the native extracellular matrix or can provide novel surface physical cues to control cellular functions.

3.4. Cytocompatibility. The live/dead fluorescence microscopy images taken after 3 days of dynamic culture on the polymeric flat surface (Figure 5a) and patterned surfaces (Figure $5 b, c$ ) indicated no visible signs of hMSCs death. In addition, cells could colonize and spread on the patterned area quite uniformly (Figure 5c). These preliminary findings suggest that the IP-Dip resin is not cytotoxic for these cells. Other studies have coated surfaces made from similar resin 

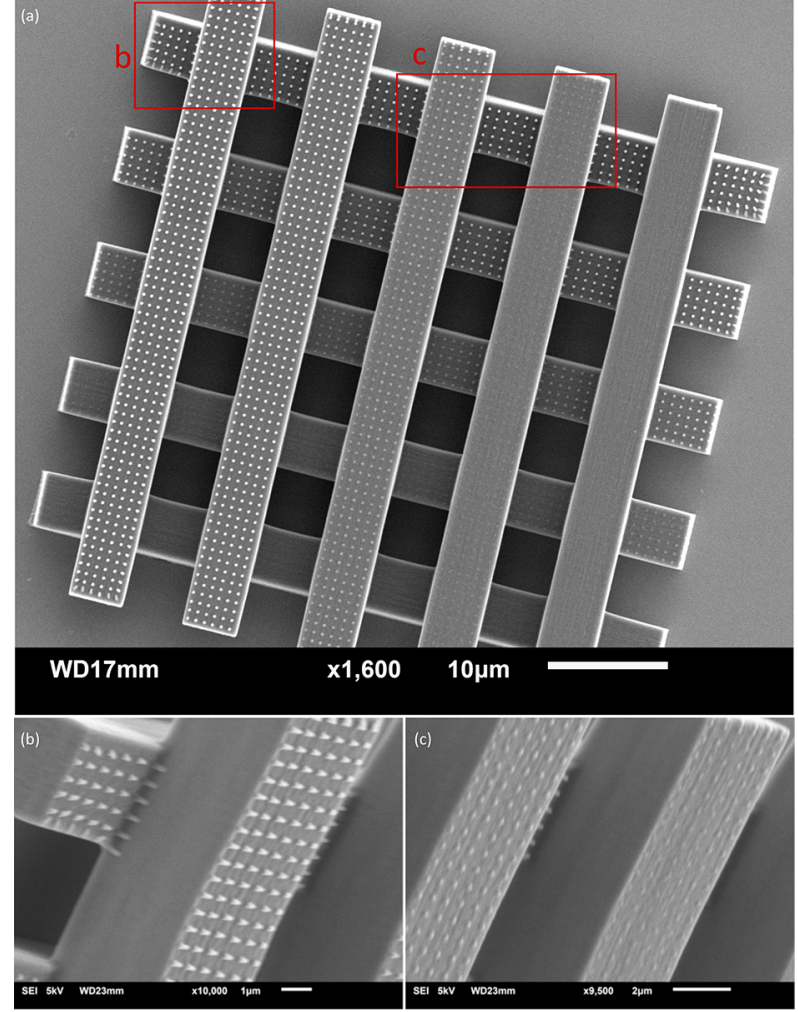

Figure 4. (a) SEM image of submicron-scale topographies incorporated into a woodpile-based microscaffold. ( $b$ and $c$ ) The submicron pillars with different heights. with biocompatible layers (e.g., $\mathrm{TiO}_{2}$, collagen, and fibronectin $)^{57-59}$ before cell culture.

A closer look at the surface-cell interface by SEM showed a well spread morphology of the hMSCs on the flat polymeric surface (Figure 5d) and visible extensions interacting with the cells from the surrounding glass substrate. The cells on the patterns (Figure 5e) exerted contractile forces on the submicron pillars, which caused them to bend and delaminate. As calculated before, the estimated cell traction force is in the order of hundreds of $\mathrm{nN}$ to a few $\mu \mathrm{N}$. It is therefore necessary to improve the pillar adhesion for cell studies. This could be achieved by increasing the diameter of the submicron pillars, reducing their height, exploring other resins, and increasing the interface distance (which starts the printing process deeper into the substrate).

\section{CONCLUSIONS}

We developed a method to create microfluidic chips decorated with surface submicron patterns as well as to fabricate $3 \mathrm{D}$ porous structures with patterned surface. Both microfluidic chips and 3D porous structures can be used in bone regeneration studies. The direct laser writing (i.e., 2PP) process has the potential of creating bone scaffolds with optimal feature sizes and a bespoke topological design. Varying the processing parameters of $2 \mathrm{PP}$ resulted in direct changes in the feature sizes at the submicron-scale. Furthermore, the created submicron topographies enhanced the surface hydrophilicity and, could withstand flow rates of up to a few $\mathrm{mL} /$ min. In addition, the results of hMSCs culture indicated no evident cytotoxicity of the material (IP-Dip resin). Although we succeeded in creating a large area of submicron patterns in
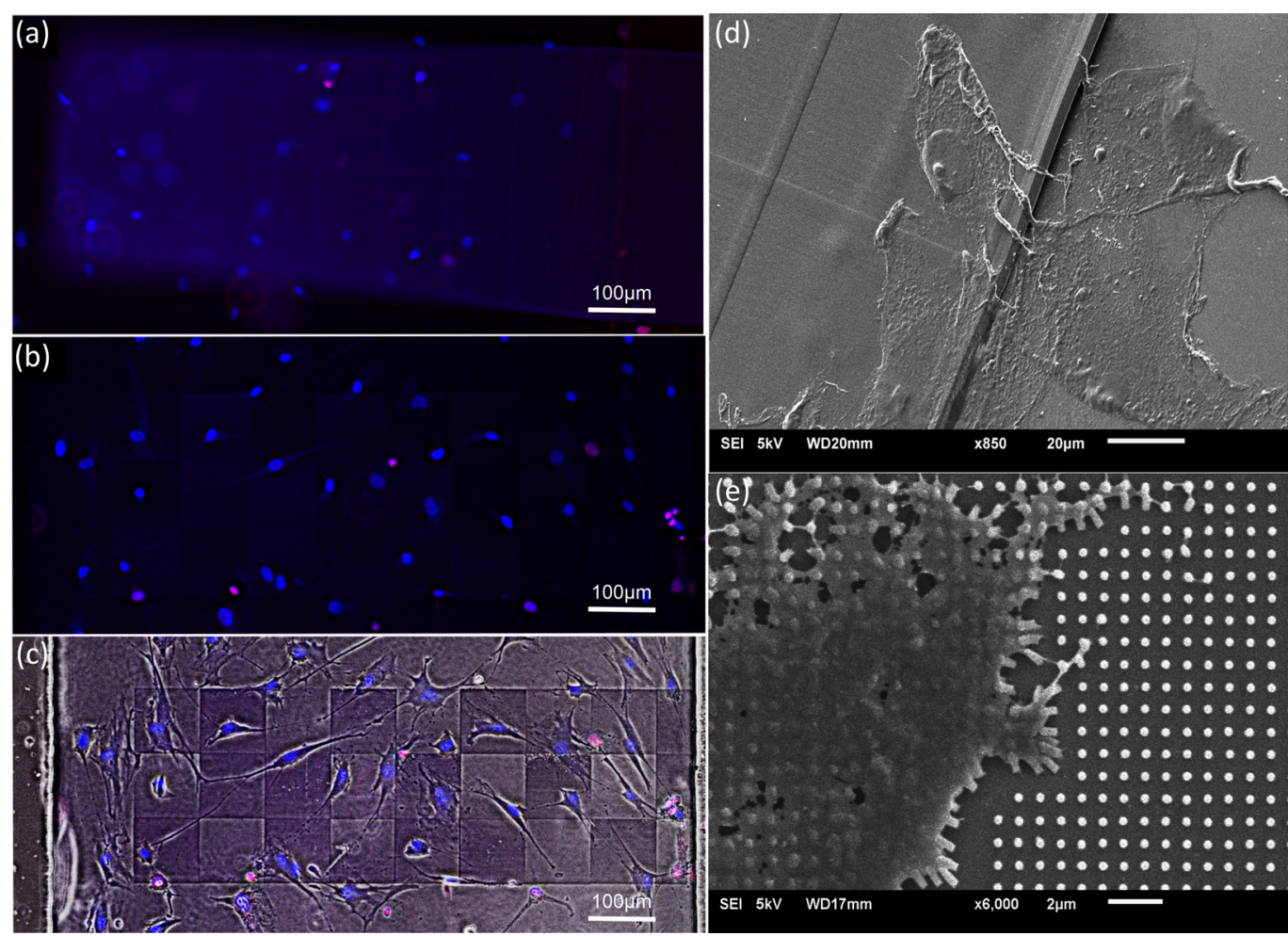

Figure 5. Live/dead staining of the hMSCs after 3 days of culture on (a) the control surface (i.e., a printed flat surface with the same material used for the submicron pillars) and (b) submicron patterned surfaces. (c) Bright-field microscopy images of the cells shown in (b). (d and e) Cellsurface interface by SEM: (d) cells residing on the flat surface and (e) cells on submicron patterned area. 
a single step, the long printing time remains a major limitation in this approach.

\section{ASSOCIATED CONTENT}

\section{S Supporting Information}

The Supporting Information is available free of charge on the ACS Publications website at DOI: 10.1021/acsbiomaterials.9b01155.

Effects of substrate types and print configurations on the submicron patterns uniformity (section S1), effects of tilt correction on the submicron patterns uniformity (section S2), effects of 2PP parameters on the dimensions and uniformity of submicron patterns (section S3), stress distribution in the anchoring surface of the submicron pillars (section S4). (PDF)

\section{AUTHOR INFORMATION}

\section{Corresponding Author}

*Tel.: +31-685267905. E-mail: m.nourigoushki@tudelft.nl; mhd.nouri71@gmail.com.

\section{ORCID}

Mahdiyeh Nouri-Goushki: 0000-0003-1665-9144

Amir A. Zadpoor: 0000-0003-3234-2112

\section{Author Contributions}

${ }^{\nabla}$ Co-first authors.

\section{Notes}

The authors declare no competing financial interest.

\section{REFERENCES}

(1) Kraus, K. H.; Kirker-Head, C. Mesenchymal stem cells and bone regeneration. Vet. Surg. 2006, 35 (3), 232-242.

(2) Giannoudis, P. V.; Dinopoulos, H.; Tsiridis, E. Bone substitutes: an update. Injury 2005, 36 (3), No. S20-S27.

(3) Li, Y.; Liu, C. Nanomaterial-based bone regeneration. Nanoscale 2017, 9 (15), 4862-4874.

(4) Henkel, J.; Woodruff, M. A.; Epari, D. R.; Steck, R.; Glatt, V.; Dickinson, I. C.; Choong, P. F.; Schuetz, M. A.; Hutmacher, D. W. Bone regeneration based on tissue engineering conceptions-a 21 st century perspective. Bone Res. 2013, 1 (3), 216.

(5) Gong, T.; Xie, J.; Liao, J.; Zhang, T.; Lin, S.; Lin, Y. Nanomaterials and bone regeneration. Bone Res. 2015, 3, 15029.

(6) Zhang, X.; Zhang, C.; Lin, Y.; Hu, P.; Shen, Y.; Wang, K.; Meng, S.; Chai, Y.; Dai, X.; Liu, X. Nanocomposite membranes enhance bone regeneration through restoring physiological electric microenvironment. ACS Nano 2016, 10 (8), 7279-7286.

(7) Sui, B.-D.; Hu, C.-H.; Liu, A.-Q.; Zheng, C.-X.; Xuan, K.; Jin, Y. Stem cell-based bone regeneration in diseased microenvironments: Challenges and solutions. Biomaterials 2019, 196, 18.

(8) Yan, Y.; Cheng, B.; Chen, K.; Cui, W.; Qi, J.; Li, X.; Deng, L. Enhanced Osteogenesis of Bone Marrow-Derived Mesenchymal Stem Cells by a Functionalized Silk Fibroin Hydrogel for Bone Defect Repair. Adv. Healthcare Mater. 2019, 8, 1801043.

(9) Yavari, S. A.; van der Stok, J.; Chai, Y. C.; Wauthle, R.; Birgani, Z. T.; Habibovic, P.; Mulier, M.; Schrooten, J.; Weinans, H.; Zadpoor, A. A. Bone regeneration performance of surface-treated porous titanium. Biomaterials 2014, 35 (24), 6172-6181.

(10) van der Stok, J.; Koolen, M.; de Maat, M.; Yavari, S. A.; Alblas, J.; Patka, P.; Verhaar, J.; van Lieshout, E.; Zadpoor, A. A.; Weinans, H. Full regeneration of segmental bone defects using porous titanium implants loaded with BMP-2 containing fibrin gels. Eur. Cells Mater. 2015, 29, 141-154.

(11) Gorgin Karaji, Z.; Speirs, M.; Dadbakhsh, S.; Kruth, J.-P.; Weinans, H.; Zadpoor, A.; Amin Yavari, S. Additively manufactured and surface biofunctionalized porous nitinol. ACS Appl. Mater. Interfaces 2017, 9 (2), 1293-1304.

(12) Dang, M.; Saunders, L.; Niu, X.; Fan, Y.; Ma, P. X. Biomimetic delivery of signals for bone tissue engineering. Bone Res. 2018, 6, 25 DOI: $10.1038 / \mathrm{s} 41413-018-0025-8$.

(13) Zadpoor, A. A. Current Trends in Metallic Orthopedic Biomaterials: From Additive Manufacturing to Bio-Functionalization, Infection Prevention, and Beyond. Int. J. Mol. Sci. 2018, 19 (9), 2684.

(14) Hohmann, J. K.; von Freymann, G. Influence of Direct Laser Written 3D Topographies on Proliferation and Differentiation of Osteoblast-Like Cells: Towards Improved Implant Surfaces. Adv. Funct. Mater. 2014, 24 (42), 6573-6580.

(15) Ahn, E. H.; Kim, Y.; An, S. S.; Afzal, J.; Lee, S.; Kwak, M.; Suh, K.-Y.; Kim, D.-H.; Levchenko, A. Spatial control of adult stem cell fate using nanotopographic cues. Biomaterials 2014, 35 (8), 2401-2410.

(16) Hammann, C.; Heerkens, C. T. H.; Hagen, C.; Zadpoor, A.; Fratila-Apachitei, L. Direct submicron patterning of titanium for bone implants. Microelectron. Eng. 2018, 195, 13-20.

(17) Dobbenga, S.; Fratila-Apachitei, L. E.; Zadpoor, A. A. Nanopattern-induced osteogenic differentiation of stem cells-A systematic review. Acta Biomater. 2016, 46, 3-14.

(18) Sjöström, T.; Dalby, M. J.; Hart, A.; Tare, R.; Oreffo, R. O.; Su, B. Fabrication of pillar-like titania nanostructures on titanium and their interactions with human skeletal stem cells. Acta Biomater. 2009, 5 (5), 1433-1441.

(19) Mirzaali, M.; Van Dongen, I.; Tümer, N.; Weinans, H.; Yavari, S. A.; Zadpoor, A. In-silico quest for bactericidal but non-cytotoxic nanopatterns. Nanotechnology 2018, 29 (43), 43LT02.

(20) Modaresifar, K.; Azizian, S.; Ganjian, M.; Fratila-Apachitei, L. E.; Zadpoor, A. A. Bactericidal effects of nanopatterns: a systematic review. Acta Biomater. 2019, 83, 29.

(21) Amin Yavari, S.; Loozen, L.; Paganelli, F. L.; Bakhshandeh, S.; Lietaert, K.; Groot, J. A.; Fluit, A. C.; Boel, C.; Alblas, J.; Vogely, H. C. Antibacterial behavior of additively manufactured porous titanium with nanotubular surfaces releasing silver ions. ACS Appl. Mater. Interfaces 2016, 8 (27), 17080-17089.

(22) Anselme, K.; Davidson, P.; Popa, A.; Giazzon, M.; Liley, M.; Ploux, L. The interaction of cells and bacteria with surfaces structured at the nanometre scale. Acta Biomater. 2010, 6 (10), 3824-3846.

(23) Ploux, L.; Anselme, K.; Dirani, A.; Ponche, A.; Soppera, O.; Roucoules, V. Opposite responses of cells and bacteria to micro/ nanopatterned surfaces prepared by pulsed plasma polymerization and UV-irradiation. Langmuir 2009, 25 (14), 8161-8169.

(24) Yang, Y.; Leong, K. W. Microfluidic platforms with nanoscale features. Microfluidic Cell Culture Systems; Elsevier: Amsterdam, 2019; pp 65-90.

(25) Chen, Y. Nanofabrication by electron beam lithography and its applications: a review. Microelectron. Eng. 2015, 135, 57-72.

(26) Cleuvenbergen, S. Direct fabrication of complex 3D hierarchical nanostructures by reactive ion etching of hollow sphere colloidal crystals. Nanoscale 2016, 8 (35), 15845-15849.

(27) Pecora, E. F.; Lawrence, N.; Gregg, P.; Trevino, J.; Artoni, P.; Irrera, A.; Priolo, F.; Dal Negro, L. Nanopatterning of silicon nanowires for enhancing visible photoluminescence. Nanoscale 2012, 4 (9), 2863-2866.

(28) Lewis, B. B.; Mound, B. A.; Srijanto, B.; Fowlkes, J. D.; Pharr, G. M.; Rack, P. D. Growth and nanomechanical characterization of nanoscale 3D architectures grown via focused electron beam induced deposition. Nanoscale 2017, 9 (42), 16349-16356.

(29) Zhang, G.; Xiao, X.; Li, B.; Gu, P.; Xue, H.; Pang, H. Transition metal oxides with one-dimensional/one-dimensional-analogue nanostructures for advanced supercapacitors. J. Mater. Chem. A 2017, 5 (18), 8155-8186.

(30) Luo, S.; Zhang, P.; Yuan, T.; Ruan, J.; Peng, C.; Pang, Y.; Sun, H.; Yang, J.; Zheng, S. Molecular self-assembly of a nanorod N-Li 4 Ti $5 \mathrm{O}$ 12/ $\mathrm{TiO} 2 / \mathrm{C}$ anode for superior lithium ion storage. J. Mater. Chem. A 2018, 6 (32), 15755-15761. 
(31) Malinauskas, M.; Farsari, M.; Piskarskas, A.; Juodkazis, S. Ultrafast laser nanostructuring of photopolymers: A decade of advances. Phys. Rep. 2013, 533 (1), 1-31.

(32) Malinauskas, M.; Zukauskas, A.; Hasegawa, S.; Hayasaki, Y.; Mizeikis, V.; Buividas, R.; Juodkazis, S. Ultrafast laser processing of materials: from science to industry. Light: Sci. Appl. 2016, 5 (8), No. e16133.

(33) Barata, D.; Provaggi, E.; van Blitterswijk, C.; Habibovic, P. Development of a microfluidic platform integrating high-resolution microstructured biomaterials to study cell-material interactions. Lab Chip 2017, 17 (23), 4134-4147.

(34) Yang, Y.; Kulangara, K.; Sia, J.; Wang, L.; Leong, K. W. Engineering of a microfluidic cell culture platform embedded with nanoscale features. Lab Chip 2011, 11 (9), 1638-1646.

(35) Bruedigam, C.; van Driel, M.; Koedam, M.; van de Peppel, J.; van der Eerden, B. C.; Eijken, M.; van Leeuwen, J. P. Basic techniques in human mesenchymal stem cell cultures: differentiation into osteogenic and adipogenic lineages, genetic perturbations, and phenotypic analyses. Curr. Protoc. Stem Cell Biol. 2011, 17 (1), 1H.3.1-1H.3.20.

(36) Sun, H.-B.; Tanaka, T.; Kawata, S. Three-dimensional focal spots related to two-photon excitation. Appl. Phys. Lett. 2002, 80 (20), 3673-3675.

(37) Wollhofen, R.; Katzmann, J.; Hrelescu, C.; Jacak, J.; Klar, T. A. $120 \mathrm{~nm}$ resolution and $55 \mathrm{~nm}$ structure size in STED-lithography. Opt. Express 2013, 21 (9), 10831-10840.

(38) Hering, J.; Waller, E. H.; Von Freymann, G. Automated aberration correction of arbitrary laser modes in high numerical aperture systems. Opt. Express 2016, 24 (25), 28500-28508.

(39) Jesacher, A.; Maurer, C.; Schwaighofer, A.; Bernet, S.; RitschMarte, M. Full phase and amplitude control of holographic optical tweezers with high efficiency. Opt. Express 2008, 16 (7), 4479-4486.

(40) Duan, H.; Yang, J. K.; Berggren, K. K. Controlled Collapse of High-Aspect-Ratio Nanostructures. Small 2011, 7 (18), 2661-2668.

(41) Shah, P. J.; Wu, Z.; Sarangan, A. M. Effects of CO2 critical point drying on nanostructured $\mathrm{SiO} 2$ thin films after liquid exposure. Thin Solid Films 2013, 527, 344-348.

(42) Zhang, Y.; Lo, C.-W.; Taylor, J. A.; Yang, S. Replica molding of high-aspect-ratio polymeric nanopillar arrays with high fidelity. Langmuir 2006, 22 (20), 8595-8601.

(43) Piao, M.-H.; Yang, D.-S.; Yoon, K.-R.; Lee, S.-H.; Choi, S.-H. Development of an electrogenerated chemiluminescence biosensor using carboxylic acid-functionalized MWCNT and Au nanoparticles. Sensors 2009, 9 (3), 1662-1677.

(44) Afre, R. A.; Hayashi, Y.; Soga, T. Enhanced photovoltaic device performance upon modification of indium tin oxide coated glass by liquid nitrogen treatment. J. Phys. D: Appl. Phys. 2009, 42 (4), 042002.

(45) Zhong, Z.; Zhong, Y.; Liu, C.; Yin, S.; Zhang, W.; Shi, D. Study on the surface wetting properties of treated indium-tin-oxide anodes for polymer electroluminescent devices. Phys. Status Solidi A 2003, 198 (1), 197-203.

(46) Besbes, S.; Ouada, H. B.; Davenas, J.; Ponsonnet, L.; Jaffrezic, N.; Alcouffe, P. Effect of surface treatment and functionalization on the ITO properties for OLEDs. Mater. Sci. Eng., C 2006, 26 (2-3), $505-510$.

(47) Quéré, D. Wetting and roughness. Annu. Rev. Mater. Res. 2008, $38,71-99$.

(48) Martines, E.; Seunarine, K.; Morgan, H.; Gadegaard, N.; Wilkinson, C. D.; Riehle, M. O. Air-trapping on biocompatible nanopatterns. Langmuir 2006, 22 (26), 11230-11233.

(49) Tunma, S.; Inthanon, K.; Chaiwong, C.; Pumchusak, J.; Wongkham, W.; Boonyawan, D. Improving the attachment and proliferation of umbilical cord mesenchymal stem cells on modified polystyrene by nitrogen-containing plasma. Cytotechnology 2013, 65 (1), 119-134.

(50) Boyan, B.; Cheng, A.; Olivares-Navarrete, R.; Schwartz, Z. Implant surface design regulates mesenchymal stem cell differentiation and maturation. Adv. Dent. Res. 2016, 28 (1), 10-17.
(51) Bruus, H. Theoretical microfluidics; Oxford University Press: Oxford, U.K., 2008; Vol. 18.

(52) Finn, R. K. Determination of the Drag on a Cylinder at Low Reynolds Numbers. J. Appl. Phys. 1953, 24 (6), 771-773.

(53) Schoen, I.; Hu, W.; Klotzsch, E.; Vogel, V. Probing cellular traction forces by micropillar arrays: contribution of substrate warping to pillar deflection. Nano Lett. 2010, 10 (5), 1823-1830.

(54) Lemma, E. D.; Rizzi, F.; Dattoma, T.; Spagnolo, B.; Sileo, L.; Qualtieri, A.; De Vittorio, M.; Pisanello, F. Mechanical properties tunability of three-dimensional polymeric structures in two-photon lithography. IEEE Trans. Nanotechnol. 2016, 16 (1), 23-31.

(55) Fu, J.; Wang, Y.-K.; Yang, M. T.; Desai, R. A.; Yu, X.; Liu, Z.; Chen, C. S. Mechanical regulation of cell function with geometrically modulated elastomeric substrates. Nat. Methods 2010, 7 (9), 733.

(56) Do Cha, H.; Hong, J. M.; Kang, T.-Y.; Jung, J. W.; Ha, D.-H.; Cho, D.-W. Effects of micro-patterns in three-dimensional scaffolds for tissue engineering applications. J. Micromech. Microeng. 2012, 22 (12), 125002.

(57) Nava, M. M.; Di Maggio, N.; Zandrini, T.; Cerullo, G.; Osellame, R.; Martin, I.; Raimondi, M. T. Synthetic niche substrates engineered via two-photon laser polymerization for the expansion of human mesenchymal stromal cells. J. Tissue Eng. Regener. Med. 2017, 11 (10), 2836-2845.

(58) Spagnolo, B.; Brunetti, V.; Leménager, G.; De Luca, E.; Sileo, L.; Pellegrino, T.; Pompa, P. P.; De Vittorio, M.; Pisanello, F. Threedimensional cage-like microscaffolds for cell invasion studies. Sci. Rep. 2015, 5, 10531.

(59) Wittig, R.; Waller, E.; von Freymann, G.; Steiner, R. Direct laser writing-mediated generation of standardized topographies for dental implant surface optimization. J. Laser Appl. 2012, 24 (4), 042011. 